\title{
Estimation of the octanol-water distribution coefficient of basic compounds by a cationic microemulsion electrokinetic chromatography system
}

Alejandro Fernández-Pumarega ${ }^{1}$, Belén Martín-Sanz ${ }^{1}$, Susana Amézqueta ${ }^{1, *}$, Elisabet Fuguet $^{1,2}$, Martí Rosés ${ }^{1, \S}$

${ }^{1}$ Departament d'Enginyeria Química i Química Analítica and Institut de Biomedicina (IBUB), Facultat de Química, Universitat de Barcelona, Martí i Franquès 1-11, 08028, Barcelona, Spain.

${ }^{2}$ Serra Húnter Programme. Generalitat de Catalunya. Spain

${ }^{\S}$ Editorial Board member

*Corresponding Author: E-mail: samezqueta@ub.edu; Tel.: (+34) 934021277; Fax: (+34) 934021233

Received: November 29, 2019; Revised: February 14, 2020; Available online: March 04, 2020

\begin{abstract}
The octanol-water partition coefficient $\left(P_{o / w}\right)$, or the octanol-water distribution coefficient $\left(D_{o / w}\right)$ for ionized compounds, is a key parameter in the drug development process. In a previous work, this parameter was estimated through the retention factor measurements in a sodium dodecyl sulfate (SDS) - microemulsion electrokinetic chromatography (MEEKC) system for acidic compounds. Nonetheless, when ionized basic compounds were analyzed, undesirable ion pairs were formed with the anionic surfactant and avoided a good estimation of $\log D_{o / w}$. For this reason, an alternative MEEKC system based on a cationic surfactant has been evaluated to estimate $P_{o / w}$ or $D_{o / w}$ of neutral compounds and ionized bases. To this end, it has been characterized through the solvation parameter model (SPM) and compared to the octanol-water partition system. Results pointed out that both systems show a similar partition behavior. Hence, the $\log P_{o / w}$ of a set of neutral compounds has been successfully correlated against the logarithm of the retention factor (log $k$ ) determined in this MEEKC system. Then, the $\log D_{o / w}$ of 6 model bases have been estimated at different $\mathrm{pH}$ values and they have been compared to data from the literature, determined by the reference shake-flask and potentiometric methods. Good agreement has been observed between the literature and the estimated values when the base is neutral or partially ionized (up to $99 \%$ of ionization).
\end{abstract}

\section{Keywords}

Lipophilicity; $\log P_{\mathrm{o} / \mathrm{w}} ; \log D_{\mathrm{o} / \mathrm{w}} ;$ MEEKC; ionized bases; solvation parameter model; microemulsion; system surrogation.

\section{Introduction}

Chromatographic systems based on different techniques such as high performance liquid chromatography (HPLC) and electrokinetic chromatography (EKC) have been widely used to determine biopartitioning properties [1-6]. In these systems, the compounds experience a partition between an aqueous phase and a stationary phase, for HPLC systems, or a pseudoestationary phase, for EKC systems, similar to the partition that compounds experience in a biological system. Therefore, if the partition in the chromatographic system and in the biological one is similar enough, it is possible to estimate the biological property through a correlation like Eq. 1: 


$$
\log S P_{\text {bio }}=\mathrm{q}+\mathrm{p} \log S P_{\text {chrom }}
$$

where $S P_{\text {bio }}$ is the solute biological property, $S P_{\text {chrom }}$ the solute physicochemical property (generally the retention factor, $k$ ), and " $q$ " and " $p$ " are the intercept and the slope of the resulting correlation between both parameters, respectively.

The lipophilicity of compounds is a property of high interest in biological processes due to its direct relationship with membrane permeability. Lipophilicity is usually evaluated through the octanol-water partition coefficient $\left(P_{\mathrm{o} / \mathrm{w}}\right)$. The direct method to determine this parameter is the shake-flask method. However, it is a tedious procedure and it is not fully automated. For this reason, the estimation of $P_{\mathrm{o} / \mathrm{w}}$ through high-throughput systems such as chromatographic ones is of great interest.

In the previous works [3-6], Eq. 1 was applied to the determination of the octanol-water partition coefficient of neutral solutes and the distribution coefficient of partially ionized acidic compounds $\left(D_{0} / \mathrm{w}\right)$ from microemulsion electrokinetic chromatography (MEEKC) measurements. A MEEKC system based on a negatively charged microemulsion (ME) constituted of heptane, 1-butanol, and sodium dodecyl sulfate (SDS) was used. The aim of the present study is to evaluate the applicability of MEEKC measurements to estimate the $\log P_{\mathrm{o} / \mathrm{w}}$ and $\log D_{\mathrm{o} / \mathrm{w}}$ of ionized basic compounds, which are positively charged in their ionized form.

However, the SDS-MEEKC system cannot be used for basic compounds since the formation of ion pairs between the surfactant, negatively charged, and the protonated base, positively charged has been observed. Thus, the retention factor would be altered leading to wrong $\log D_{\mathrm{o} / \mathrm{w}}$ estimated values. In this work, the replacement of the anionic surfactant (SDS) by a cationic one (tetradecyltrimethylammonium bromide, TTAB) is tested to solve this problem. So, as the only role of the surfactant is to stabilize the oil droplets, we think that the TTAB-MEEKC system should be able to estimate the $P_{\mathrm{o} / \mathrm{w}}$ or $D_{\mathrm{o} / \mathrm{w}}$ of basic solutes as well as the SDS-MEEKC does for acidic solutes. Actually, Ishihama et al. [7] evaluated 3 different surfactants and showed that neither the ionic groups nor the hydrocarbon chain lengths affected the selectivity of the ME for neutral compounds. Thus, the aim of the present work is to test this potential ability of the TTAB-MEEKC system.

\section{Theory}

The retention factor of an ionized base can be calculated through Eq. 2 [8]:

$$
k=\frac{\mu-\left(\frac{\mu}{\mu_{0}}\right)_{\text {ephedrine cation }} \cdot \mu_{0}}{\mu_{\mathrm{ME}}-\mu}
$$

where, $\mu$ and $\mu_{0}$ are the electrophoretic mobility of the compound in MEEKC and in plain buffer (capillary zone electrophoresis, $\mathrm{CZE}$ ), respectively. $\mu_{M E}$ is the electrophoretic mobility of the ME marker in the MEEKC analysis, and $\left(\mu / \mu_{0}\right)_{\text {ephedrine cation }}$ is the viscosity correction factor (whose value is 0.84 ). This correction needs to be introduced since two solutions with quite different viscosities $(\eta)$ are employed in $k$ calculation, and $\mu$ is inversely related to $\eta$ [9]. Therefore, the mobility in CZE is corrected to make it equivalent to the one measured in MEEKC. Ephedrine has been selected because it is a small and polar compound that is not retained in the chromatographic system when it is fully ionized. So, $\left(\mu / \mu_{0}\right)_{\text {ephedrine cation }}$ results from the ratio of mobilities of the ephedrine cation measured in MEEKC and CZE modes.

$\mu$ values can be obtained from:

$$
\mu=\left[\frac{1}{t_{\mathrm{r}}}-\frac{1}{t_{0}}\right] \cdot\left[\frac{L_{\mathrm{T}} L_{\mathrm{D}}}{V}\right]
$$


where, $t_{\mathrm{r}}$ and $t_{0}$ are the elution times of the compound and the electroosmotic flow marker, respectively. $L_{T}$ and $L_{D}$ are the total and the effective length of the capillary, respectively, and $V$ is the voltage applied during the separation.

Similarly to acidic solutes [10], the retention factor of a monoprotic basic compound in a MEEKC system varies with the $\mathrm{pH}$ of the media through:

$$
k=\frac{k_{(\mathrm{BH}}^{+}+k_{(\mathrm{B})} \cdot 10^{\mathrm{pH}-\mathrm{p} K_{\mathrm{a}}^{\prime}}}{1+10^{\mathrm{pH}-\mathrm{p} K_{\mathrm{a}}^{\prime}}}
$$

where $k_{(\mathrm{B})}$ and $k_{(\mathrm{BH}+)}$ are the retention factors of the neutral and the fully ionized base, respectively, and the $\mathrm{p} K_{\mathrm{a}}{ }^{\prime}$ is the apparent acidity constant of the base.

\section{Experimental}

\section{Equipment}

All the analysis were performed with a capillary electrophoresis (CE) 7100 equipped with a UV diode array detector from Agilent technologies (Santa Clara, CA, USA). The fused-silica capillaries employed were from Polymicro Technologies (Phoenix, AZ, USA). The capillaries used had a $50 \mu \mathrm{m}$ internal diameter.

Water was purified by a Milli-Q plus system from Millipore (Burlington, MA, USA) with a resistivity of 18.2 $\mathrm{M} \Omega \cdot \mathrm{cm}$. To determine the $\mathrm{pH}$ of the solutions a $\mathrm{pH}$-meter GLP 22 from Crison (Barcelona, Spain) was used.

\section{Reagents}

Hydrochloric acid (1N Tritisol $\left.^{\mathrm{TM}}\right)$, and sodium hydroxide $\left(0.5 \mathrm{~N}\right.$ Tritisol $\left.{ }^{\mathrm{TM}}\right)$ were acquired from Merck (Darmstadt, Germany). Methanol (HPLC-grade) was obtained from Thermo Fisher Scientific (Waltham, MA, USA). Heptane (99\%), dodecanophenone (98\%), TTAB (>99\%), 1-butanol ( $\geq 99.7 \%), 2$-[Bis(2-hydroxyethyl)amino]-2-(hydroxymethyl)propane-1,3diol (Bis-tris) (>99\%), and sodium phosphate dodecahydrate (>98 \%) were purchased from Sigma-Aldrich (St. Louis, MO, USA). Disodium hydrogen phosphate (99.5\%) was from Baker (Phillipsburg, NJ, USA).

Test compounds with high purities were supplied from different manufacturers: Sigma-Aldrich, Merck, Baker, Carlo Erba (Milan, Italy), Fluka (St. Louis, MO, USA), Riedel-de Häen (Seelze, Germany), and Scharlab (Barcelona, Spain).

\section{Analysis conditions}

\section{Buffer preparation}

To prepare the buffers at $\mathrm{pH} 5.4$ and $\mathrm{pH} 7.0$, the $\mathrm{pH}$ of an aliquot of a $0.2 \mathrm{M}$ bis-tris solution previously protonated with $\mathrm{HCl}$ was adjusted with $\mathrm{NaOH}$, while to prepare the buffer at $\mathrm{pH} 11.4$ a mixture of a $0.2 \mathrm{M}$ disodium hydrogen phosphate solution and a $0.2 \mathrm{M}$ sodium phosphate dodecahydrate solution was used. All the buffers were prepared maintaining the ionic strength at $0.05 \mathrm{M}$.

\section{ME preparation}

To prepare the ME 1.70, g of TTAB were dissolved in around $70 \mathrm{~mL}$ of the corresponding buffer solution. Then, $8.15 \mathrm{~mL}$ of 1-butanol were added, finishing by the addition of $1.15 \mathrm{~mL}$ of heptane. The co-surfactant and the oil were added under continuous magnetic stirring, and if the solution remained turbid, it was sonicated until clarification [4]. Finally, buffer was added up to a total volume of $100 \mathrm{~mL}$. The concentrations of each component with respect the total volume of the ME were: $1.70 \%(w / v)$ TTAB, 
$8.15 \%(v / v)$ 1-butanol, and $1.15 \%(v / v)$ heptane.

\section{$\underline{\text { Instrumental parameters }}$}

All the measurements were performed keeping the temperature at $25{ }^{\circ} \mathrm{C}$ and using a fused-silica capillary with an effective length of $30 \mathrm{~cm}$ and a total length of $38.5 \mathrm{~cm}$. The voltage applied in MEEKC measurements was negative and between -11.5 and $-14 \mathrm{kV}$. Regarding the internal pressure applied, it ranged between 0 and 25 mbar. The analysis conditions were different, depending on the $\mathrm{pH}$, to obtain the best electrophoretic window possible. In the case of CZE measurements, the analyses were performed employing positive voltages.

Most compounds were dissolved at $200 \mathrm{mg} \cdot \mathrm{L}^{-1}$ in a 9:1 ME:methanol mixture in the MEEKC analysis and in a 9:1 buffer:methanol mixture in the CZE analysis. Solutes with low UV absorbance were analyzed at a concentration of $10 \%(\mathrm{v} / \mathrm{v})$.

The injection of the compounds was performed applying during $5 \mathrm{~s}$ an internal pressure of $50 \mathrm{mbar}$. The detection of the solutes was performed at $\lambda=200,214$ or $254 \mathrm{~nm}$ (depending on the absorbance profile of the compounds). The ME marker used was dodecanophenone (200 $\mathrm{mg} \cdot \mathrm{L}^{-1}$, detected at $\lambda=254 \mathrm{~nm}$ ), while the electroosmotic flow marker was methanol $(10 \% \mathrm{v} / \mathrm{v}$, detected at $\lambda=200 \mathrm{~nm})$ [11].

\section{Comparison of systems characterized by the solvation parameter model}

The TTAB-MEEKC system has been characterized through the solvation parameter model (SPM) to check its suitability to surrogate the octanol-water partition system [12]. SPM relates a free energy solvation property (in this work the $\log P_{\mathrm{o} / \mathrm{w}}$ or the $\log k$ ) to five different solute descriptors. The equation resulting from the characterization of a system through the SPM model is shown below:

$$
\log S P=c+e E+s S+a A+b B+v V
$$

where SP is the solute property in a given partitioning system; $E, S, A, B$ and $V$ are the Abraham solute descriptors (which are specific for each compound), and the coefficients $e, s, a, b$ and $v$ provide the characteristics of the partition system. $E$ is the excess molar refraction, $S$ the solute dipolarity/polarizability, $A$ and $B$ are the solute hydrogen-bond acidity and basicity descriptors, respectively, and $V$ the McGowan's volume of the molecule. Each of the system coefficients are complementary to their respective solute descriptor. The system coefficients can be calculated by a multiple linear regression between the depending solute property of a set of representative neutral compounds and their solute descriptors [13].

The similarity between different systems can be determined by comparing the coefficients resulting from the characterization of the systems by the SPM. Lazaro, et al. [14] proposed the distance parameter $d$ to compare two different systems ( $i$ and $j$ ) through their normalized SPM coefficients (Eqs. 6-10).

$$
\begin{aligned}
& e_{\mathrm{u}}=\frac{e}{l} \\
& s_{\mathrm{u}}=\frac{s}{l} \\
& a_{\mathrm{u}}=\frac{a}{l} \\
& b_{\mathrm{u}}=\frac{b}{l} \\
& v_{\mathrm{u}}=\frac{v}{l}
\end{aligned}
$$

where $e_{\mathrm{u}}, s_{\mathrm{u}}, a_{\mathrm{u}}, b_{\mathrm{u}}, v_{\mathrm{u}}$ are the normalized coefficients (or coefficients of the unitary vector) and $l$ is the length of the coefficients vector; which is calculated as follows: 


$$
l=\sqrt{e^{2}+s^{2}+a^{2}+b^{2}+v^{2}}
$$

then, the $d$ parameter is calculated by Eq. 12 .

$$
d=\sqrt{\left(e_{\mathrm{ui}}-e_{\mathrm{uj}}\right)^{2}+\left(s_{\mathrm{ui}}-s_{\mathrm{uj}}\right)^{2}+\left(a_{\mathrm{ui}}-a_{\mathrm{uj}}\right)^{2}+\left(b_{\mathrm{ui}}-b_{\mathrm{uj}}\right)^{2}+\left(v_{\mathrm{ui}}-v_{\mathrm{uj}}\right)^{2}}
$$

The smaller the $d$ parameter, the more similar will be the systems compared. As a practical convention, if the distance between the systems compared is below 0.25 , it can be concluded that these systems are analogous [14].

Moreover, the precision of the correlation (Eq. 13) between $\log P_{\mathrm{o} / \mathrm{w}}$ and $\log k$ in the TTAB-MEEKC system has been estimated following the approach published elsewhere [15].

The overall precision of the correlation $\left(S D_{\text {corr }}{ }^{2}\right)$ can be estimated by the sum of three different contributions:

$$
S D_{\text {corr }}^{2}=S D_{P_{\mathrm{o} / \mathrm{w}}}^{2}+\left(\mathrm{p} \cdot S D_{\mathrm{MEEKC}}\right)^{2}+S D_{\mathrm{d}}^{2}
$$

$S D_{\mathrm{Po} / \mathrm{w}}$ and $S D_{\mathrm{MEEKC}}$ are the standard deviations (SD) obtained from the characterization through the SPM of the octanol-water partition system and the TTAB-MEEKC system, respectively. $S D_{\mathrm{d}}$ and " $\mathrm{p}$ " are the SD and the slope obtained from the correlation of the $\log P_{\mathrm{o} / \mathrm{w}}$ and $\log k$ values of a set of representative solutes. The values of the parameters were calculated using the equations resulting from the SPM characterization and the descriptors of the solutes. By using these equations, $S D_{\text {Po/w }}$ and $S D_{\text {MEEKC }}$ are equal to 0 . So, the SD of this correlation can be attributed uniquely to the dissimilarity between the systems.

\section{Data analysis}

Table Curve 2D from Systat Software Inc. (San Jose, CA, USA) was employed to obtain the log $D_{\mathrm{o} / \mathrm{w}}-\mathrm{pH}$ profiles of the basic compounds. Data calculations were performed using Excel 2010 from Microsoft (Redmond, WA, USA).

\section{Results and Discussion}

Suitability of the TTAB-MEEKC system to estimate $\log P_{o / w}$ values

\section{Characterization of the MEEKC system by the SPM}

The TTAB-MEEKC system has been characterized through the SPM in order to later evaluate its similarity with the octanol-water partition system. To obtain accurate solvation coefficients of the model, representative analytes have been selected to characterize the system. In 2001, Fuguet et al. [13] proposed 71 compounds from a 2975 solute data base, selected according to their solute-solvent interactions, as a good set to characterize chromatographic systems by the SPM.

The retention factors of the proposed set of compounds in the TTAB-MEEKC system have been measured at $\mathrm{pH}$ 7.0, where all compounds are neutral. During the characterization, some of the compounds have been discarded since they are not lipophilic enough and elute with the EOF marker (propan-1,3-diol, butan-1,4-diol, and pentan-1,5-diol), they are too lipophilic and they co-elute with the ME marker (butylbenzene, and $\alpha$-pinene), or they present other experimental troubles in the TTAB-MEEKC system (myrcene, pentan-1-ol and pentan-3-ol). The retention factor of neutral compounds can be calculated using Eq. 2, being $\mu_{o}$ equal to 0 as they are uncharged. Table 1 lists the compounds used in the characterization together with their solute descriptors, and the $\log k$ in the TTAB-MEEKC system. Moreover, the $\log P_{o / w}$ values of each compound reported in the literature are also listed [16-30]. 
Table 1. Abraham solute descriptors, $\log k$ and $\log P_{o / w}$ of the chosen neutral analytes

\begin{tabular}{|c|c|c|c|c|c|c|c|}
\hline Compound & $E$ & $S$ & $A$ & $B$ & $v$ & $\log k$ & $\log P_{\mathrm{o} / \mathrm{w}}{ }^{\mathrm{a}}$ \\
\hline Propan-1-ol & 0.236 & 0.42 & 0.37 & 0.48 & 0.5900 & -0.71 & 0.30 \\
\hline Propan-2-ol & 0.212 & 0.36 & 0.33 & 0.56 & 0.5900 & -0.79 & 0.05 \\
\hline Butan-1-ol & 0.224 & 0.42 & 0.37 & 0.48 & 0.7309 & 0.08 & 0.88 \\
\hline Pentan-1-ol & 0.219 & 0.42 & 0.37 & 0.48 & 0.8718 & - & 1.56 \\
\hline Pentan-3-ol & 0.218 & 0.36 & 0.33 & 0.56 & 0.8718 & - & 1.21 \\
\hline Propan-1,3-diol & 0.397 & 0.91 & 0.77 & 0.85 & 0.6487 & - & -1.04 \\
\hline Butan-1,4-diol & 0.395 & 0.93 & 0.72 & 0.90 & 0.7860 & - & -0.83 \\
\hline Pentan-1,5-diol & 0.388 & 0.95 & 0.72 & 0.91 & 0.9305 & - & -0.43 \\
\hline Thiourea & 0.840 & 0.82 & 0.77 & 0.87 & 0.5696 & -1.06 & -1.02 \\
\hline Benzene & 0.610 & 0.52 & 0.00 & 0.14 & 0.7164 & 0.36 & 2.13 \\
\hline Toluene & 0.601 & 0.52 & 0.00 & 0.14 & 0.8573 & 0.77 & 2.69 \\
\hline Ethylbenzene & 0.613 & 0.51 & 0.00 & 0.15 & 0.9982 & 1.10 & 3.15 \\
\hline Propylbenzene & 0.604 & 0.50 & 0.00 & 0.15 & 1.1391 & 1.53 & 3.68 \\
\hline Butylbenzene & 0.600 & 0.51 & 0.00 & 0.15 & 1.2800 & - & 4.38 \\
\hline$p$-Xylene & 0.613 & 0.52 & 0.00 & 0.16 & 0.9982 & 1.12 & 3.15 \\
\hline Naphthalene & 1.340 & 0.92 & 0.00 & 0.20 & 1.0854 & 1.21 & 3.37 \\
\hline Chlorobenzene & 0.718 & 0.65 & 0.00 & 0.07 & 0.8388 & 0.85 & 2.90 \\
\hline Bromobenzene & 0.882 & 0.73 & 0.00 & 0.09 & 0.8914 & 0.99 & 2.99 \\
\hline Anisole & 0.708 & 0.75 & 0.00 & 0.29 & 0.9160 & 0.28 & 2.11 \\
\hline Benzaldehyde & 0.820 & 1.00 & 0.00 & 0.39 & 0.8730 & -0.10 & 1.47 \\
\hline Acetophenone & 0.818 & 1.01 & 0.00 & 0.48 & 1.0139 & -0.03 & 1.58 \\
\hline Propiophenone & 0.804 & 0.95 & 0.00 & 0.51 & 1.1548 & 0.35 & 2.24 \\
\hline Butyrophenone & 0.797 & 0.95 & 0.00 & 0.51 & 1.2957 & 0.68 & 2.65 \\
\hline Valerophenone & 0.795 & 0.95 & 0.00 & 0.50 & 1.4366 & 1.05 & 3.40 \\
\hline Heptanophenone & 0.720 & 0.95 & 0.00 & 0.50 & 1.7184 & 1.88 & 4.41 \\
\hline Benzophenone & 1.447 & 1.50 & 0.00 & 0.50 & 1.4808 & 1.02 & 3.18 \\
\hline Methyl benzoate & 0.733 & 0.85 & 0.00 & 0.46 & 1.0726 & 0.35 & 2.12 \\
\hline Benzyl benzoate & 1.264 & 1.42 & 0.00 & 0.51 & 1.6804 & 1.49 & 3.97 \\
\hline Benzonitrile & 0.742 & 1.11 & 0.00 & 0.33 & 0.8711 & -0.06 & 1.56 \\
\hline Aniline & 0.955 & 0.96 & 0.26 & 0.50 & 0.8162 & -0.28 & 0.90 \\
\hline o-Toluidine & 0.970 & 0.90 & 0.23 & 0.59 & 0.9751 & -0.04 & 1.32 \\
\hline 3-Chloroaniline & 1.050 & 1.10 & 0.30 & 0.36 & 0.9390 & 0.47 & 1.88 \\
\hline 4-Chloroaniline & 1.060 & 1.10 & 0.30 & 0.35 & 0.9390 & 0.41 & 1.84 \\
\hline 2-Nitroaniline & 1.180 & 1.37 & 0.30 & 0.36 & 0.9904 & 0.25 & 1.79 \\
\hline 3-Nitroaniline & 1.200 & 1.71 & 0.40 & 0.35 & 0.9904 & 0.07 & 1.32 \\
\hline 4-Nitroaniline & 1.220 & 1.91 & 0.42 & 0.38 & 0.9904 & 0.11 & 1.39 \\
\hline Nitrobenzene & 0.871 & 1.11 & 0.00 & 0.28 & 0.8906 & 0.16 & 1.85 \\
\hline 2-Nitroanisole & 0.965 & 1.34 & 0.00 & 0.38 & 1.0902 & 0.17 & 1.73 \\
\hline Benzamide & 0.990 & 1.50 & 0.49 & 0.67 & 0.9728 & -0.39 & 0.64 \\
\hline 4-Aminobenzamide & 1.340 & 1.94 & 0.80 & 0.94 & 1.0726 & -0.86 & -0.41 \\
\hline Acetanilide & 0.870 & 1.36 & 0.46 & 0.69 & 1.1137 & -0.17 & 1.19 \\
\hline 4-Chloroacetanilide & 0.980 & 1.50 & 0.64 & 0.51 & 1.2357 & 0.51 & 2.12 \\
\hline Phenol & 0.805 & 0.89 & 0.60 & 0.30 & 0.7751 & 0.05 & 1.48 \\
\hline 3-Methylphenol & 0.822 & 0.88 & 0.57 & 0.34 & 0.9160 & 0.38 & 2.02 \\
\hline
\end{tabular}


Table 1. Continued...

\begin{tabular}{|c|c|c|c|c|c|c|c|}
\hline Compound & $E$ & $S$ & $A$ & $B$ & $V$ & $\log k$ & $\log P_{\mathrm{o} / \mathrm{w}}{ }^{\mathrm{a})}$ \\
\hline 2,3-Dimethylphenol & 0.850 & 0.90 & 0.52 & 0.36 & 1.0569 & 0.64 & - \\
\hline 2,4-Dimethylphenol & 0.840 & 0.80 & 0.53 & 0.39 & 1.0569 & 0.71 & 2.42 \\
\hline Thymol & 0.822 & 0.79 & 0.52 & 0.44 & 1.3387 & 1.20 & 3.34 \\
\hline 4-Chlorophenol & 0.915 & 1.08 & 0.67 & 0.20 & 0.8975 & 0.79 & 2.39 \\
\hline Catechol & 0.970 & 1.10 & 0.88 & 0.47 & 0.8338 & -0.09 & 0.88 \\
\hline Resorcinol & 0.980 & 1.00 & 1.10 & 0.58 & 0.8338 & -0.18 & 0.80 \\
\hline Hydroquinone & 1.000 & 1.00 & 1.16 & 0.60 & 0.8338 & -0.43 & 0.58 \\
\hline 2-Naphthol & 1.520 & 1.08 & 0.61 & 0.40 & 1.1441 & 1.04 & 2.84 \\
\hline 1,2,3-Trihydroxybenzene & 1.165 & 1.35 & 1.35 & 0.62 & 0.8925 & -0.29 & - \\
\hline Furan & 0.369 & 0.53 & 0.00 & 0.13 & 0.5363 & -0.31 & 1.34 \\
\hline 2,3-Benzofuran & 0.888 & 0.83 & 0.00 & 0.15 & 0.9053 & 0.71 & 2.67 \\
\hline Quinoline & 1.268 & 0.97 & 0.00 & 0.51 & 1.0443 & 0.20 & 2.15 \\
\hline Pyrrole & 0.613 & 0.73 & 0.41 & 0.29 & 0.5774 & -0.44 & 0.75 \\
\hline Pyrimidine & 0.606 & 1.00 & 0.00 & 0.65 & 0.6342 & -1.03 & -0.40 \\
\hline Antipyrine & 1.320 & 1.50 & 0.00 & 1.48 & 1.5502 & -0.67 & 0.56 \\
\hline Caffeine & 1.500 & 1.60 & 0.00 & 1.33 & 1.3632 & -0.77 & -0.01 \\
\hline Corticosterone & 1.860 & 3.43 & 0.40 & 1.63 & 2.7389 & 0.65 & 1.90 \\
\hline Cortisone & 1.960 & 3.50 & 0.36 & 1.87 & 2.7546 & 0.23 & 1.47 \\
\hline Hydrocortisone & 2.030 & 3.49 & 0.71 & 1.90 & 2.7975 & 0.39 & 1.53 \\
\hline Estradiol & 1.800 & 3.30 & 0.88 & 0.95 & 2.1988 & 1.35 & 4.01 \\
\hline Estratriol & 2.000 & 3.36 & 1.40 & 1.22 & 2.2575 & 0.67 & 2.45 \\
\hline Monuron & 1.140 & 1.50 & 0.47 & 0.78 & 1.4768 & 0.32 & 1.94 \\
\hline Myrcene & 0.483 & 0.29 & 0.00 & 0.21 & 1.3886 & - & 4.17 \\
\hline$\alpha$-Pinene & 0.446 & 0.14 & 0.00 & 0.12 & 1.2574 & - & 4.83 \\
\hline Geraniol & 0.513 & 0.63 & 0.39 & 0.66 & 1.4903 & 1.07 & - \\
\hline
\end{tabular}

a) From references [16-30].

To obtain the SPM coefficients of the TTAB-MEEKC system, butan-1-ol, and thiourea were considered as outliers and discarded, as they presented standard residues higher than 2.5. Estradiol had a standard residue slightly greater than 2.5 . However, it was not considered as outlier because discarding it had no big influence on the result.

The parameters and statistics ( $F$, Fisher's F parameter; SD, standard deviation; $R^{2}$, determination coefficient; $n$, number of compounds) resulting from the system characterization are:

$\log k=-0.96( \pm 0.05)+0.44( \pm 0.08) E-0.63( \pm 0.06) S+0.20( \pm 0.05) A-2.13( \pm 0.07) B+2.36( \pm 0.06) V(14)$ $R^{2}=0.972, S D=0.12, F=368, n=59$.

The large values of the coefficients $b$ and $v$ show that the hydrogen bond basicity and the volume of the solute are the more important parameters. The $v$ coefficient is positive, meaning that it is easier to form a cavity to place the solute in the ME phase than in the aqueous one. A negative $b$ coefficient is obtained indicating a higher hydrogen bonding acidity of the aqueous phase with respect to the ME phase. The $e$ coefficient is positive, so the ME is more polarizable than the aqueous phase. The negative value of the $s$ coefficient indicates that the ME system is less dipolar than the aqueous phase. The coefficient $a$ is close to zero, showing that hydrogen bonding basicities of $\mathrm{ME}$ and aqueous phases are similar, and the variation of solute hydrogen bond acidity has a small effect on the system. 


\section{$\underline{\text { Model comparison }}$}

The TTAB-MEEKC system has been compared to the octanol-water partition system and the SDS-MEEKC system. The last system was used in other research studies to estimate the $\log P_{\mathrm{o} / \mathrm{w}}$ of ionizable acids $[5,6]$.

The normalized coefficients obtained from the characterization of the TTAB-MEEKC, the octanol-water partition [31], and the SDS-MEEKC [3] (calculated using Eqs. 6-11), and the $d$ distance parameter (Eq. 12) are summarized in Table 2.

Table 2. Normalized coefficients and $d$ distances of the compared systems.

\begin{tabular}{cccccccccccc}
\hline System & $\boldsymbol{e}_{\mathrm{u}}$ & $\boldsymbol{s}_{\mathrm{u}}$ & $\boldsymbol{a}_{\mathrm{u}}$ & $\boldsymbol{b}_{\mathrm{u}}$ & $\boldsymbol{v}_{\mathbf{u}}$ & $\mathbf{n}$ & $\boldsymbol{S D}$ & $\mathbf{R}^{\mathbf{2}}$ & $\boldsymbol{d}$ & Ref. \\
\hline TTAB MEEKC & 0.13 & -0.19 & 0.06 & -0.65 & 0.72 & 59 & 0.12 & 0.972 & - & This work \\
Octanol-water & 0.11 & -0.20 & 0.01 & -0.65 & 0.72 & 613 & 0.12 & 0.994 & 0.06 & {$[31]$} \\
SDS MEEKC & 0.07 & -0.16 & -0.01 & -0.67 & 0.72 & 53 & 0.09 & 0.988 & 0.11 & {$[3]$} \\
\hline
\end{tabular}

The normalized coefficients of the three systems are very similar, being for all of them the $b$ and $v$ coefficients the most important ones. Furthermore, the $d$ parameter between TTAB-MEEKC and octanolwater partition is very small (0.06), meaning that the TTAB-MEEKC system is a good approximation to surrogate the octanol-water partition one, and consequently, lipophilicity. The $d$ parameter obtained between TTAB-MEEKC and SDS-MEEKC is also small (0.11), therefore TTAB is a good substitute of SDS for $\log P_{\mathrm{o} / \mathrm{w}}$ determination.

The chromatographic precision of the correlation between log $k$ in the TTAB-ME and the octanol-water partition has also been calculated. As explained before and elsewhere in the literature [15], the overall precision results can be estimated from a sum of three different contributions $\left(S D_{\text {Po/w }}{ }^{2},\left(p \cdot S D_{\text {MEEKC }}\right)^{2}, S D_{d}{ }^{2}\right)$. Here, the largest contribution to the overall precision comes from the chromatographic data $\left(\left(\mathrm{p} \cdot S D_{\text {MEEKC }}\right)^{2}=\right.$ $0.034)$ and the dissimilarity between the two systems $\left(S D_{d}{ }^{2}=0.013\right)$ is similar than the variance of the biopartitioning data $\left(S D_{P_{0} / w}{ }^{2}=0.013\right)$. However, the calculated overall precision $\left(S D_{\text {corr }}{ }^{2}=0.061\right)$ is low. Therefore, the correlation obtained between the TTAB-MEEKC and octanol-water systems should be good, meaning that, the TTAB-MEEKC system is a good candidate to estimate satisfactorily the octanol/water lipophilicity of neutral compounds.

\section{Correlation between $\log P_{\mathrm{o} / \mathrm{w}}$ and $\log k$}

A linear correlation between the $\log P_{\mathrm{o} / \mathrm{w}}$ and the $\log k$ of 58 solutes presented in Table 1 has been established. The correlation between $\log P_{\mathrm{o} / \mathrm{w}}$ and $\log k$ is plotted in Figure 1 . The equation and statistics obtained are as follows:

$$
\begin{aligned}
& \log P_{\mathrm{o} / \mathrm{w}}=1.68( \pm 0.05) \cdot \log k+1.35( \pm 0.04) \\
& \mathrm{R}^{2}=0.954 ; \mathrm{SD}=0.25 ; \mathrm{F}=1174 ; \mathrm{n}=58 ;
\end{aligned}
$$

which is not significantly different from the $\log P_{\mathrm{o} / \mathrm{w}}-\log k$ correlation already reported for the SDS-MEEKC system employing a set of neutral compounds (Eq. 16) [6].

$$
\begin{aligned}
& \log P_{\mathrm{o} / \mathrm{w}}=1.60( \pm 0.11) \cdot \log k+1.51( \pm 0.08) \\
& \mathrm{R}^{2}=0.916 ; \mathrm{SD}=0.33 ; \mathrm{F}=196 ; \mathrm{n}=20
\end{aligned}
$$

Good statistics have been obtained for the correlation: $R^{2}$ value close to 1 , and a small SD value of the regression, whereby the TTAB-MEEKC system has been proved to provide a good estimation of the $\log P_{\mathrm{o} / \mathrm{w}}$ of neutral compounds, as already pointed out through the model comparison tools. Therefore, Eq. 15 is going to be used as calibration curve for the estimation of $D_{\mathrm{o} / \mathrm{w}}$ of ionized bases in the following section. 


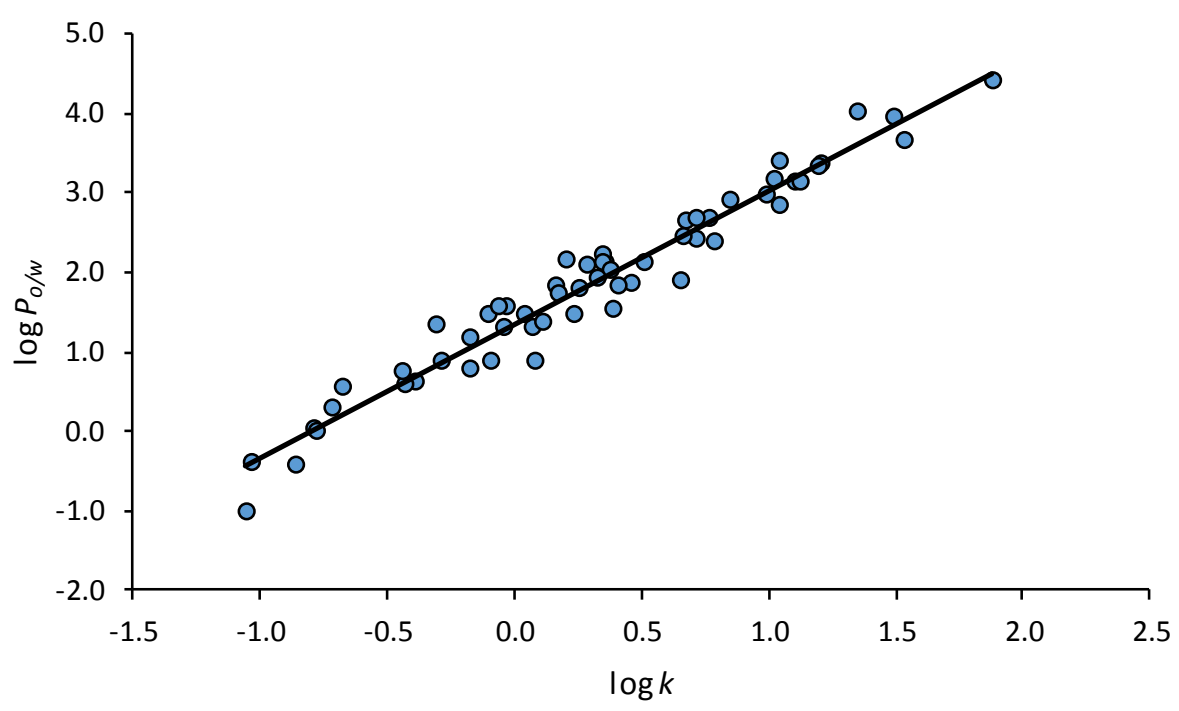

Figure 1. $\log P_{\mathrm{o} / \mathrm{w}}$ vs log $k$ correlation with the compounds from Table 1 (Eq. 15).

$\log D_{o / w}$ estimation of ionized basic compounds.

With the aim to extent the applicability of the method to ionizable bases, the $\log D_{\mathrm{o} / \mathrm{w}}$ of six basic compounds (alprenolol, nadolol, oxprenolol, penbutolol, pindolol, and propranolol) have been estimated at different $\mathrm{pH}$ values, thus at different ionization degrees and compared to literature experimental values. All these compounds are used as pharmaceutical drugs and present a basic group with a $\mathrm{p} K_{\mathrm{a}}$ inside the electrophoretic $\mathrm{pH}$ working range (2-12) [27].

Similarly to $k$ (Eq. 4), the $\log D_{\mathrm{o} / \mathrm{w}}$ of ionized bases depends on the $\mathrm{p} K_{\mathrm{a}}{ }^{\prime}$ of the base and the $\mathrm{pH}$ of the ME through:

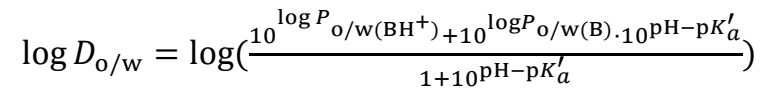

where $\log P_{\mathrm{o} / \mathrm{w}(\mathrm{BH}+)}$ and $\log P_{\mathrm{o} / \mathrm{w}(\mathrm{B})}$ are the logarithms of the octanol-water partition coefficient of the fully ionized and neutral base, respectively. The $\log D_{\mathrm{o} / \mathrm{w}}-\mathrm{pH}$ profiles for each of the model compounds have been calculated fitting Eq. 17 to the $\log D_{\mathrm{o} / \mathrm{w}}$ values determined at different values of $\mathrm{pH}$ taken from the literature [32-37]. The $\log D_{\mathrm{o} / \mathrm{w}}$ values compiled have been determined using the reference shake-flask, and the $\mathrm{pH}$-metric titration methods. The profiles, the parameters and the statistics resulting from these fittings can be found in Figure 2 and Table 3.

Table 3. Parameters resulting from the fit of Eq. 17 to $\log D_{\mathrm{o} / \mathrm{w}} \mathrm{vs} \mathrm{pH}$ data. $k_{(\mathrm{BH}+)}$ and $k_{(\mathrm{B})}$ have been experimentally determined in the TTAB-MEEKC system.

\begin{tabular}{ccccccccc}
\hline Compound & $\mathbf{p} \boldsymbol{K}_{\mathrm{a}}{ }^{\prime}(\mathrm{SD})$ & $\log \boldsymbol{P}_{\mathrm{o} / \mathrm{w}(\mathrm{BH}+)}(\mathbf{S D})$ & $\log \boldsymbol{P}_{\mathrm{o} / \mathrm{w}(\mathrm{B})}(\mathbf{S D})$ & $\mathbf{R}^{2}$ & $\mathbf{S D}$ & $\mathbf{F}$ & $\boldsymbol{k}_{(\mathrm{BH}+)}$ & $\boldsymbol{k}_{(\mathrm{B})}$ \\
\hline Alprenolol & $9.63(0.28)$ & $-0.46(0.16)$ & $3.20(0.23)$ & 0.939 & 0.36 & 116 & 0.67 & 11.09 \\
Nadolol & $9.31(0.21)$ & $-2.05(0.11)$ & $0.83(0.15)$ & 0.975 & 0.21 & 156 & 0.05 & 0.88 \\
Oxprenolol & $9.38(0.16)$ & $-1.43(0.10)$ & $2.14(0.13)$ & 0.985 & 0.18 & 328 & 0.14 & 2.82 \\
Penbutolol & $9.37(0.31)$ & $1.23(0.24)$ & $4.06(0.24)$ & 0.987 & 0.24 & 37 & 3.20 & 61.64 \\
Pindolol & $9.32(0.17)$ & $-1.39(0.10)$ & $1.79(0.13)$ & 0.970 & 0.23 & 243 & 0.16 & 2.43 \\
Propranolol & $9.24(0.26)$ & $-0.17(0.15)$ & $2.99(0.21)$ & 0.908 & 0.37 & 94 & 1.19 & 16.51 \\
\hline
\end{tabular}

Then, the $k_{(\mathrm{BH}+)}$ and $k_{(\mathrm{B})}$ of the bases have been determined in the TTAB-MEEKC system at $\mathrm{pH} 5.4$ and 11.4, respectively (Table 3). The retention factors and the $\mathrm{p} K_{\mathrm{a}}^{\prime}$ of each of the bases have been used in Eq. 4 to estimate $k$ values at the $\mathrm{pH}$ of interest. Then, $\log D_{\mathrm{o} / \mathrm{w}}$ has been estimated through Eq. 15. The $\log D_{\mathrm{o} / \mathrm{w}}$ from the literature [32-37] and the estimated values are summarized in Table 4. The ionization degree 
$\left(\alpha_{(\mathrm{BH}+)}\right)$ of the bases at each $\mathrm{pH}$ value (calculated using Eq. 18) are also provided.

$$
\alpha_{\left(\mathrm{BH}^{+}\right)}=\frac{10^{\mathrm{p} K_{\mathrm{a}}^{\prime}-\mathrm{pH}}}{1+10^{\mathrm{p} K_{\mathrm{a}}^{\prime}-\mathrm{pH}}}
$$
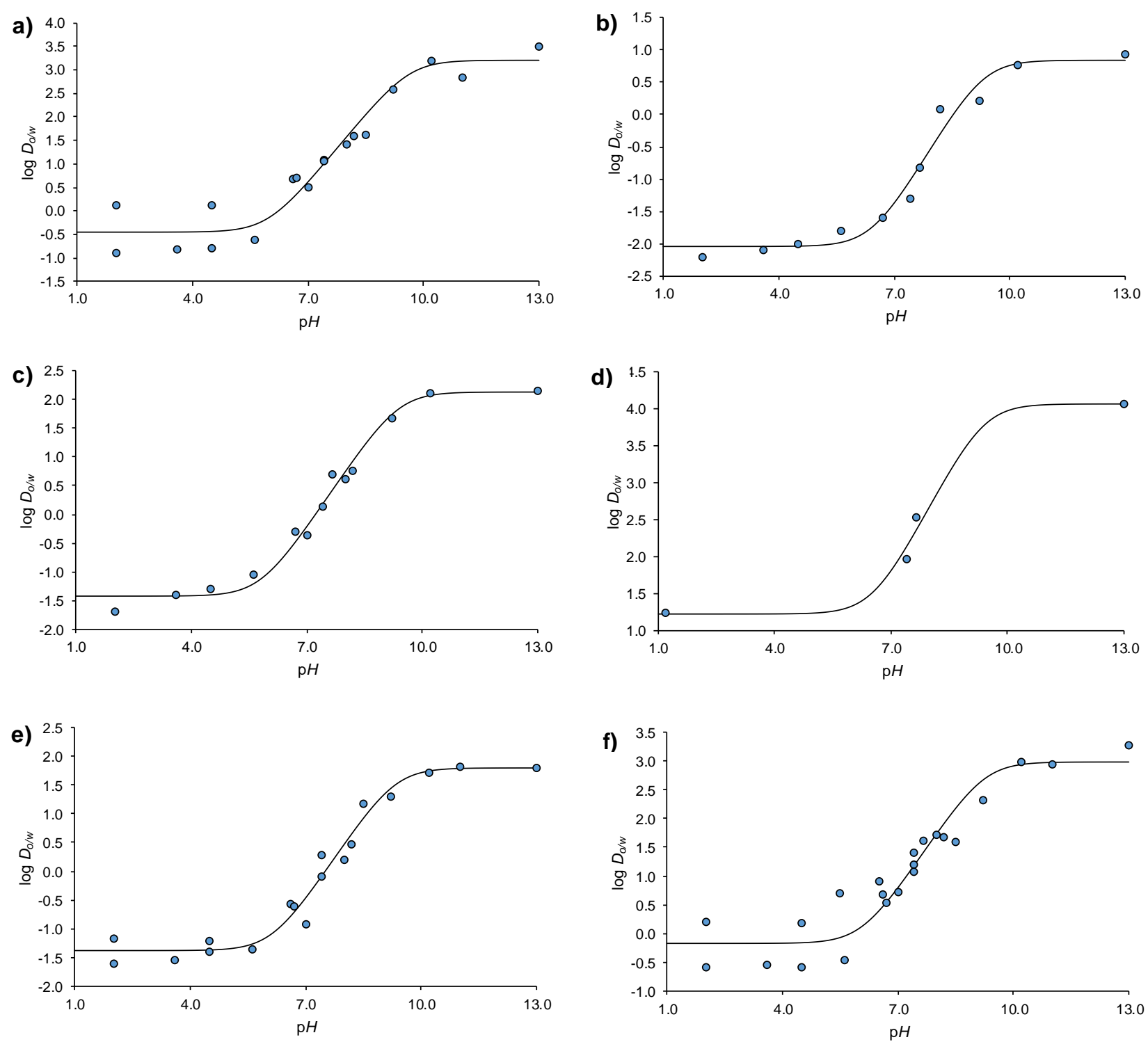

Figure 2. $\log D_{\mathrm{o} / \mathrm{w}}-\mathrm{pH}$ profiles obtained adjusting Eq. 17 to the data from the literature $(\bullet):$ a) alprenolol, b) nadolol, c) oxprenolol, d) penbutolol, e) pindolol, f) propranolol.

Figure 3 compares the estimated and the experimental data from Table 4 for bases neutral or fully ionized (Figure 3A) or partially ionized, from 1 to $99 \%$ (Figure 3B). The same figure also represents a line with a slope of 1 and an intercept of 0 , and two extra lines showing the $95 \%$ confidence interval ( \pm 2 SD) of the calibration curve for neutral compounds.

Analyzing the results from Figure 3 and Table 4, it can be concluded that, generally, accurate estimated $\log D_{\mathrm{o} / \mathrm{w}}$ values are obtained especially when the compound is in its neutral or partially ionized forms. The error obtained is similar to the one obtained for neutral compounds, as indicated by the error bars. Nonetheless, when the bases are highly or totally ionized $(\alpha \approx 1)$ an overestimation of the parameter is observed. Note that when the compound is completely ionized the results are less comparable due to the high dependence of the $\log P_{\mathrm{o} / \mathrm{w}(\mathrm{BH}+)}$ value with the solution medium (nature of counter-ions and concentrations as usually ion pairs are formed). 
The results obtained in this work agree with the ones published before for acidic compounds when a SDS-MEEKC system was employed [6].

Table 4. Differences between literature $\left(\log D_{\text {lit. }}\right)$ and estimated $\left(\log D_{\text {est. }}\right)$ values of the bases at different $\mathrm{pH}$ values through Eq. 15.

\begin{tabular}{|c|c|c|c|c|c|}
\hline Compound & $\mathrm{pH}$ & $\alpha$ & $\log D_{\text {lit. }}^{\text {a) }}$ & $\log D_{\text {est. }}$ & $\log D_{\text {lit. }}-\log D_{e s t}$ \\
\hline \multirow{18}{*}{ Alprenolol } & 2.0 & 1.00 & 0.13 & 1.06 & -0.93 \\
\hline & 2.0 & 1.00 & -0.89 & 1.06 & -1.95 \\
\hline & 3.6 & 1.00 & -0.80 & 1.06 & -1.86 \\
\hline & 4.5 & 1.00 & 0.14 & 1.06 & -0.92 \\
\hline & 4.5 & 1.00 & -0.77 & 1.06 & -1.83 \\
\hline & 5.6 & 1.00 & -0.60 & 1.06 & -1.66 \\
\hline & 6.6 & 1.00 & 0.68 & 1.07 & -0.39 \\
\hline & 6.7 & 1.00 & 0.73 & 1.07 & -0.34 \\
\hline & 7.0 & 1.00 & 0.51 & 1.08 & -0.57 \\
\hline & 7.4 & 0.99 & 1.09 & 1.12 & -0.03 \\
\hline & 7.4 & 0.99 & 1.08 & 1.12 & -0.04 \\
\hline & 8.0 & 0.98 & 1.44 & 1.28 & 0.16 \\
\hline & 8.2 & 0.96 & 1.60 & 1.38 & 0.22 \\
\hline & 8.5 & 0.93 & 1.63 & 1.59 & 0.04 \\
\hline & 9.2 & 0.73 & 2.59 & 2.26 & 0.33 \\
\hline & 10.2 & 0.21 & 3.20 & 2.94 & 0.26 \\
\hline & 11.0 & 0.04 & 2.84 & 3.08 & -0.24 \\
\hline & 13.0 & 0.00 & 3.50 & 3.11 & 0.39 \\
\hline \multirow{11}{*}{ Nadolol } & 2.0 & 1.00 & -2.20 & -0.84 & -1.36 \\
\hline & 3.6 & 1.00 & -2.10 & -0.84 & -1.26 \\
\hline & 4.5 & 1.00 & -2.00 & -0.84 & -1.16 \\
\hline & 5.6 & 1.00 & -1.80 & -0.83 & -0.97 \\
\hline & 6.7 & 1.00 & -1.60 & -0.81 & -0.79 \\
\hline & 7.4 & 0.99 & -1.30 & -0.70 & -0.60 \\
\hline & 7.65 & 0.98 & -0.82 & -0.61 & -0.21 \\
\hline & 8.2 & 0.93 & 0.08 & -0.26 & 0.34 \\
\hline & 9.2 & 0.56 & 0.22 & 0.70 & -0.48 \\
\hline & 10.2 & 0.11 & 0.76 & 1.17 & -0.41 \\
\hline & 13.0 & 0.00 & 0.93 & 1.26 & -0.33 \\
\hline \multirow{13}{*}{ Oxprenolol } & 2.0 & 1.00 & -1.70 & -0.08 & -1.62 \\
\hline & 3.6 & 1.00 & -1.40 & -0.08 & -1.32 \\
\hline & 4.5 & 1.00 & -1.30 & -0.08 & -1.22 \\
\hline & 5.6 & 1.00 & -1.04 & -0.08 & -0.96 \\
\hline & 6.7 & 1.00 & -0.30 & -0.06 & -0.24 \\
\hline & 7.0 & 1.00 & -0.37 & -0.03 & -0.34 \\
\hline & 7.4 & 0.99 & 0.13 & 0.05 & 0.08 \\
\hline & 7.65 & 0.98 & 0.69 & 0.13 & 0.56 \\
\hline & 8.0 & 0.96 & 0.61 & 0.33 & 0.28 \\
\hline & 8.2 & 0.94 & 0.76 & 0.49 & 0.27 \\
\hline & 9.2 & 0.60 & 1.67 & 1.49 & 0.18 \\
\hline & 10.2 & 0.13 & 2.10 & 2.01 & 0.09 \\
\hline & 13.0 & 0.00 & 2.16 & 2.11 & 0.05 \\
\hline
\end{tabular}


Table 4. Continued...

\begin{tabular}{|c|c|c|c|c|c|}
\hline Compound & $\mathrm{pH}$ & $\alpha$ & $\log D_{\text {lit. }}^{\text {a) }}$ & $\log D_{\text {est. }}$ & $\log D_{\text {lit. }}-\log D_{\text {est. }}$ \\
\hline \multirow{4}{*}{ Penbutolol } & 1.2 & 1.00 & 1.24 & 2.20 & -0.96 \\
\hline & 7.4 & 0.99 & 1.97 & 2.33 & -0.36 \\
\hline & 7.65 & 0.98 & 2.53 & 2.41 & 0.12 \\
\hline & 13.0 & 0.00 & 4.06 & 4.36 & -0.30 \\
\hline \multirow{18}{*}{ Pindolol } & 2.0 & 1.00 & -1.17 & 0.01 & -1.18 \\
\hline & 2.0 & 1.00 & -1.60 & 0.01 & -1.61 \\
\hline & 3.6 & 1.00 & -1.55 & 0.01 & -1.56 \\
\hline & 4.5 & 1.00 & -1.40 & 0.01 & -1.41 \\
\hline & 4.5 & 1.00 & -1.22 & 0.01 & -1.23 \\
\hline & 5.6 & 1.00 & -1.35 & 0.01 & -1.36 \\
\hline & 6.6 & 1.00 & -0.56 & 0.03 & -0.59 \\
\hline & 6.7 & 1.00 & -0.62 & 0.04 & -0.66 \\
\hline & 7.0 & 1.00 & -0.92 & 0.06 & -0.98 \\
\hline & 7.4 & 0.99 & 0.29 & 0.13 & 0.16 \\
\hline & 7.4 & 0.99 & -0.10 & 0.13 & -0.23 \\
\hline & 8.0 & 0.95 & 0.19 & 0.38 & -0.19 \\
\hline & 8.2 & 0.93 & 0.46 & 0.52 & -0.06 \\
\hline & 8.5 & 0.87 & 1.18 & 0.78 & 0.40 \\
\hline & 9.2 & 0.57 & 1.29 & 1.45 & -0.16 \\
\hline & 10.2 & 0.12 & 1.72 & 1.91 & -0.19 \\
\hline & 11.0 & 0.02 & 1.82 & 1.98 & -0.16 \\
\hline & 13.0 & 0.00 & 1.80 & 2.00 & -0.20 \\
\hline \multirow{22}{*}{ Propranolol } & 2.0 & 1.00 & 0.20 & 1.48 & -1.28 \\
\hline & 2.0 & 1.00 & -0.58 & 1.48 & -2.06 \\
\hline & 3.6 & 1.00 & -0.55 & 1.48 & -2.03 \\
\hline & 4.5 & 1.00 & 0.18 & 1.48 & -1.30 \\
\hline & 4.5 & 1.00 & -0.59 & 1.48 & -2.07 \\
\hline & 5.5 & 1.00 & 0.7 & 1.5 & -0.8 \\
\hline & 5.6 & 1.00 & -0.46 & 1.48 & -1.94 \\
\hline & 6.5 & 1.00 & 0.9 & 1.50 & -0.6 \\
\hline & 6.6 & 1.00 & 0.68 & 1.50 & -0.82 \\
\hline & 6.7 & 1.00 & 0.54 & 1.50 & -0.96 \\
\hline & 7.0 & 0.99 & 0.73 & 1.53 & -0.80 \\
\hline & 7.4 & 0.99 & 1.4 & 1.6 & -0.2 \\
\hline & 7.4 & 0.99 & 1.20 & 1.60 & -0.40 \\
\hline & 7.4 & 0.99 & 1.07 & 1.60 & -0.53 \\
\hline & 7.65 & 0.97 & 1.62 & 1.68 & -0.06 \\
\hline & 8.0 & 0.95 & 1.72 & 1.86 & -0.14 \\
\hline & 8.2 & 0.92 & 1.67 & 2.01 & -0.34 \\
\hline & 8.5 & 0.85 & 1.60 & 2.27 & -0.67 \\
\hline & 9.2 & 0.52 & 2.32 & 2.91 & -0.59 \\
\hline & 10.2 & 0.10 & 2.98 & 3.33 & -0.35 \\
\hline & 11.0 & 0.02 & 2.94 & 3.38 & -0.44 \\
\hline & 13.0 & 0.00 & 3.28 & 3.40 & -0.12 \\
\hline
\end{tabular}

a) From references [32-37]. 

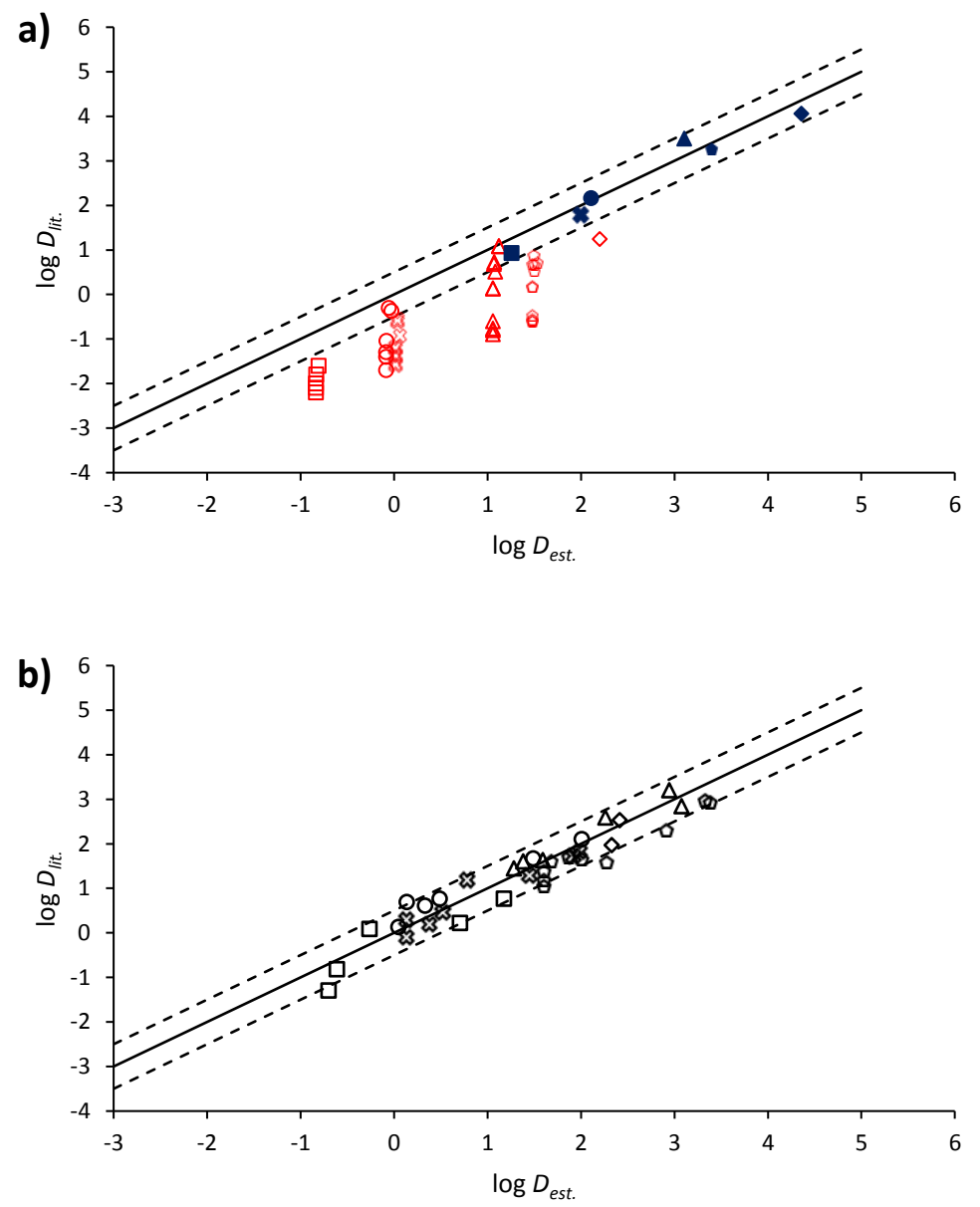

Figure 3. Correlation between the $\log D_{\mathrm{o} / \mathrm{w}}$ from the literature $\left(\log D_{\text {lit. }}\right)$ and the $\log D_{\mathrm{o} / \mathrm{w}}$ estimated through the present method (log $D_{\text {est. }}$ ) at different degrees of ionization. Alprenolol ( $\boldsymbol{\Delta})$, nadolol $(\boldsymbol{\square})$, oxprenolol $(\bullet)$, penbutolol $(\boldsymbol{\vee})$, pindolol $(\boldsymbol{*})$, and propranolol (•). a) Comparison of the data when the bases are in their neutral form (0-1 \% of ionization, blue full symbol) or fully ionized ( $\geq 99 \%$ of ionization, red empty symbol). b) Comparison of the data when the bases are partially ionized (1-99\% of ionization). In addition, a line with a slope of 1 and an intercept of 0 , and two extra lines corresponding to \pm 2 SD of the calibration curve (Eq. 15) (which corresponds to the 95 $\%$ confidence interval) are also shown.

\section{Conclusions}

The TTAB-MEEKC system characterized through the solvation parameter model shows great similarity with the octanol-water partition and the SDS-MEEKC systems. Thus, both ME-based systems can be used for $\log P_{\mathrm{o} / \mathrm{w}}$ estimation of neutral solutes.

A good correlation has been obtained between $\log P_{\mathrm{o} / \mathrm{w}}$ data and $\log \mathrm{k}$ measured in the TTAB-MEEKC system for neutral compounds. Moreover, the applicability of the method has been widen to the estimation of $\log D_{\mathrm{o} / \mathrm{w}}$ of partially ionized bases. Accurate estimated values have been obtained when the compound is neutral or partially ionized (up to a $99 \%$ of ionization).

Acknowledgements: Financial support from the Ministerio de Economía y Competitividad from the Spanish Government (CTQ2017-88179-P) and the Catalan Government (2017SGR1074) is acknowledged. AFP wishes to thank the University of Barcelona for his APIF PhD fellowship.

Conflict of interest: The authors declare no conflict of interest.

\section{References}

[1] A. Fernández-Pumarega, S. Amézqueta, S. Farré, L. Muñoz-Pascual, M.H. Abraham, E. Fuguet, M. Rosés. Modeling aquatic toxicity through chromatographic systems. Analytical Chemistry 89 (2017) 7996-8003.

[2] S. Soriano-Meseguer, E. Fuguet, A. Port, M. Rosés. Estimation of skin permeation by liquid chromatography. ADMET \& DMPK 6 (2018) 140-152.

[3] M.H. Abraham, C. Treiner, M. Roses, C. Ràfols, Y. Ishihama. Linear free energy relationship analysis of microemulsion electrokinetic chromatographic determination of lipophilicity. Journal of Chromatography A 752 (1996) 243-249. 
[4] X. Subirats, H.P. Yuan, V. Chaves, N. Marzal, M. Rosés. Microemulsion electrokinetic chromatography as a suitable tool for lipophilicity determination of acidic, neutral, and basic compounds. Electrophoresis 37 (2016) 2010-2016.

[5] A. Fernández-Pumarega, S. Amézqueta, E. Fuguet, M. Rosés. Feasibility of the estimation of octanolwater distribution coefficients of acidic drugs by microemulsion electrokinetic chromatography. ADMET \& DMPK 6 (2018) 55-60.

[6] A. Fernández-Pumarega, S. Amézqueta, E. Fuguet, M. Rosés. Estimation of the octanol-water distribution coefficient of acidic compounds by microemulsion electrokinetic chromatography. Journal of pharmaceutical and biomedical analysis 179 (2020) 112981.

[7] Y. Ishihama, Y. Oda, N. Asakawa. Hydrophobicity of cationic solutes measured by electrokinetic chromatography with cationic microemulsions. Analytical Chemistry 68 (1996) 4281-4284.

[8] A. Fernández-Pumarega, S. Amézqueta, E. Fuguet, M. Rosés. Determination of the retention factor of ionizable compounds in microemulsion electrokinetic chromatography. Analytica Chimica Acta 1078 (2019) 221-230.

[9] D.R. Baker, Capillary electrophoresis, Wiley, Hoboken, NJ, USA, 1995, p. 39.

[10] M.G. Khaledi, S.C. Smith, J.K. Strasters. Micellar electrokinetic capillary chromatography of acidic solutes: migration behavior and optimization strategies. Analytical Chemistry 63 (1991) 1820-1830.

[11] E. Fuguet, C. Ràfols, E. Bosch, M. Rosés. Solute-solvent interactions in micellar electrokinetic chromatography: IV. Characterization of electroosmotic flow and micellar markers. Electrophoresis 23 (2002) 56-66.

[12] M.H. Abraham. Scales of solute hydrogen-bonding: their construction and application to physicochemical and biochemical processes. Chemical Society Reviews 22 (1993) 73-83.

[13] E. Fuguet, C. Ràfols, E. Bosch, M.H. Abraham, M. Rosés. Solute-solvent interactions in micellar electrokinetic chromatography: III. Characterization of the selectivity of micellar electrokinetic chromatography systems. Journal of Chromatography A 942 (2002) 237-248.

[14] E. Lázaro, C. Ràfols, M.H. Abraham, M. Rosés. Chromatographic estimation of drug disposition properties by means of immobilized artificial membranes (IAM) and C18 columns. Journal of Medicinal Chemistry 49 (2006) 4861-4870.

[15] M. Hidalgo-Rodríguez, E. Fuguet, C. Ràfols, M. Rosés. Estimation of biological properties by means of chromatographic systems: evaluation of the factors that contribute to the variance of biologicalchromatographic correlations. Analytical Chemistry 82 (2010) 10236-10245.

[16] E.O. Dillingham, R.W. Mast, G.E. Bass, J. Autian. Toxicity of methyl- and halogen-substituted alcohols in tissue culture relative to structure-activity models and acute toxicity in mice. Journal of Pharmaceutical Sciences 62 (1973) 22-30.

[17] Bio-Loom, BioByte Corp. (Claremont, CA, USA), http://www.biobyte.com Version 1.7. (Accessed 25 October 2019).

[18] T. Sotomatsu, M. Shigemura, Y. Murata, T. Fujita. Octanol/water partition coefficient of orthosubstituted aromatic solutes. Journal of Pharmaceutical Sciences 82 (1993) 776-781.

[19] S. Banerjee, S.H. Yalkowsky, C. Valvani. Water solubility and octanol/water partition coefficients of organics. Limitations of the solubility-partition coefficient correlation. Environmental Science and Technology 14 (1980) 1227-1229.

[20] J. Li, E.M. Perdue, S.G. Pavlostathis, R. Araujo. Physicochemical properties of selected monoterpenes. Environment International 24 (1998) 353-358.

[21] P.R. Rich, R. Harper. Partition coefficients of quinones and hydroquinones and their relation to biochemical reactivity. FEBS Letters 269 (1990) 139-144.

[22] D.E. Leszczynski, R.M. Schafer. Nonspecific and metabolic interactions between steroid hormones and human plasma lipoproteins. Lipids 25 (1990) 711-718.

[23] D.M. Miller. Evidence that interfacial transport is rate-limiting during passive cell membrane permeation. Biochimica et Biophysica Acta (BBA) - Biomembranes 1065 (1991) 75-81. 
[24] T. Fujita, J. Iwasa, C. Hansch. A new substituent constant, $\pi$, derived from partition coefficients. Journal of the American Chemical Society 86 (1964) 5175-5180.

[25] J. Iwasa, T. Fujita, C. Hansch. Substituent constants for aliphatic functions obtained from partition coefficients. Journal of Medicinal Chemistry 8 (1965) 150-153.

[26] J. De Bruijn, F. Busser, W. Seinen, J. Hermens. Determination of octanol/water partition coefficients for hydrophobic organic chemicals with the "slow-stirring" method. Environmental Toxicology and Chemistry 8 (1989) 499-512.

[27] A. Avdeef. Absorption and Drug Development: Solubility, Permeability, and Charge State, 2nd edition, Wiley, Hoboken, NJ, USA, 2012, p. 108-126.

[28] A. Andrés, M. Rosés, C. Ràfols, E. Bosch, S. Espinosa, V. Segarra, J.M. Huerta. Setup and validation of shake-flask procedures for the determination of partition coefficients $(\log D)$ from low drug amounts. European Journal of Pharmaceutical Sciences 76 (2015) 181-191.

[29] L. Hitzel, A.P. Watt, K.L. Locker. An increased throughput method for the determination of partition coefficients. Pharmaceutical Research 17 (2000) 1389-1395.

[30] C. Hansch, S.M. Anderson. The effect of intramolecular hydrophobic bonding on partition coefficients. Journal of Organic Chemistry 32 (1967) 2583-2586.

[31] M.H. Abraham, H.S. Chadha, G.S. Whiting, R.C. Mitchell. Hydrogen bonding. 32. An analysis of wateroctanol and water-alkane partitioning and the $\Delta \log$ P parameter of seiler. Journal of Pharmaceutical Sciences 83 (1994) 1085-1100.

[32] S. Winiwarter, N.M. Bonham, F. Ax, A. Hallberg, H. Lennernäs, A. Karlén. Correlation of human jejunal permeability (in vivo) of drugs with experimentally and theoretically derived parameters. A multivariate data analysis approach. Journal of Medicinal Chemistry 41 (1998) 4939-4949.

[33] F. Barbato, G. Caliendo, M.I. La Rotonda, P. Morrica, C. Silipo, A. Vittoria. Relationships between octanol-water partition data, chromatographic indices and their dependence on $\mathrm{pH}$ in a set of betaadrenoceptor blocking agents. Farmaco 45 (1990) 647-663.

[34] M. Recanatini. Partition and distribution coefficients of aryloxypropanolamine $\beta$-adrenoceptor antagonists. Journal of Pharmacy and Pharmacology 44 (1992) 68-70.

[35] H.S. Huang, R.D. Schoenwald, J.L. Lach. Corneal penetration behavior of $\beta$-blocking agents II: assessment of barrier contributions. Journal of Pharmaceutical Sciences 72 (1983) 1272-1279.

[36] D. Hellenbrecht, B. Lemmer, G. Wiethold, H. Grobecker. Measurement of hydrophobicity, surface activity, local anaesthesia, and myocardial conduction velocity as quantitative parameters of the nonspecific membrane affinity of nine $\beta$-adrenergic blocking agents. Naunyn-Schmiedeberg's Archives of Pharmacology 277 (1973) 211-226.

[37] N. Gulyaeva, A. Zaslavsky, P. Lechner, M. Chlenov, A. Chait, B. Zaslavsky. Relative hydrophobicity and lipophilicity of $\beta$-blockers and related compounds as measured by aqueous two-phase partitioning, octanol - buffer partitioning, and HPLC. European Journal of Pharmaceutical Sciences 17 (2002) 8193.

(C)2020 by the authors; licensee IAPC, Zagreb, Croatia. This article is an open-access article distributed under the terms and conditions of the Creative Commons Attribution license (http://creativecommons.org/licenses/by/3.0/) (cc) $\mathrm{EY}$ 